\title{
The Relative Contribution of Physical Fitness to the Technical Execution Score in Youth Rhythmic Gymnastics
}

\author{
by \\ Olyvia Donti ${ }^{1}$, Gregory C. Bogdanis ${ }^{1}$, Maria Kritikou ${ }^{1}$, Anastasia Donti ${ }^{1}$, \\ Kalliopi Theodorakou ${ }^{1}$
}

This study examined the association between physical fitness and a technical execution score in rhythmic gymnasts varying in the performance level. Forty-six young rhythmic gymnasts (age: $9.9 \pm 1.3$ years) were divided into two groups (qualifiers, $n=24$ and non-qualifiers, $n=22$ ) based on the results of the National Championships. Gymnasts underwent a series of physical fitness tests and technical execution was evaluated in a routine without apparatus. There were significant differences between qualifiers and non-qualifiers in the technical execution score $(p=0.01, d=1.0)$, shoulder flexion ( $p=0.01, d=0.8$ ), straight leg raise ( $p=0.004, d=0.9$ ), sideways leg extension ( $p=0.002, d=0.9$ ) and body fat ( $p=.021, d=0.7$ ), but no differences were found in muscular endurance and jumping performance. The technical execution score for the non-qualifiers was significantly correlated with shoulder extension $(r=0.423, p<0.05)$, sideways leg extension ( $r=0.687, p<0.01)$, push ups $(r=0.437, p<0.05)$ and body fat $(r=0.642, p<0.01)$, while there was only one significant correlation with sideways leg extension $(r=0.467, p<0.05)$ for the qualifiers. Multiple regression analysis revealed that sideways leg extension, body fat, and push ups accounted for a large part (62.9\%) of the variance in the technical execution score for the non-qualifiers, while for the qualifiers, only $37.3 \%$ of the variance in the technical execution score was accounted for by sideways leg extension and spine flexibility. In conclusion, flexibility and body composition can effectively discriminate between qualifiers and non-qualifiers in youth rhythmic gymnastics. At the lower level of performance (non-qualifiers), physical fitness seems to have a greater effect on the technical execution score.

Key words: flexibility, body composition, gymnastics.

\section{Introduction}

The relationship between the competition score and physical fitness variables in rhythmic gymnastics has been examined in a number of previous studies (Bobo-Arce and Mendez-Rial, 2013; Hume et al., 1993; Rutkauskaite and Skarbalius, 2009). Anthropometric variables such as body composition, the arm span and mid-thigh circumference, have been suggested as significant determinants of the rhythmic gymnastics competition score (Douda et al., 2008; PurenovicIvanovic and Popovic, 2014). Physical fitness variables such as flexibility, explosive strength and aerobic capacity have also been identified as contributing factors to performance (Di Cagno et al., 2009; Douda et al., 2008; Rutkauskaite and Skarbalius, 2009; 2011). However, several anthropometric variables that are identified as determinants of performance such as the arm span, leg length, and height, cannot be altered by training and mainly depend on the initial and long-term selection processes across ages (Karpenko, 2003). Regarding physical fitness components that are trainable, previous studies have reported contradictory results. For example

1 - Faculty of Physical Education and Sport Science, University of Athens, Greece. 
Hume et al. (1993) reported moderate but significant correlations between age, lean body mass, flexibility, leg power, motor proficiency and the final score (the average final score of 4 competitions) in rhythmic gymnasts aged 7-27 years. Furthermore, in another study, aerobic capacity was identified as the most important predictor of the final competition score in 34 elite and non-elite junior rhythmic gymnasts (Douda et al., 2008). In that study, $92.5 \%$ of the reported variance of competitive performance was explained by aerobic capacity $(58.9 \%)$, the arm span $(12 \%)$, midthigh circumference $(13.1 \%)$ and body mass $(8.5 \%)$. Although aerobic capacity is important for recovery between strenuous efforts in all sports (Bogdanis, 2012), other physical abilities such as flexibility, balance and explosive strength may be more associated with competitive performance in rhythmic gymnastics and in particular at young age. Therefore, there is a need to precisely define sport specific competitive demands and identify the physical fitness variables that can be improved in the training process and are more important for the developing athlete.

One explanation for the discrepancies observed in the literature regarding the contribution of physical and anthropometric variables to performance is the definition of the "performance score" used in the regression analyses. For example, some studies use the final score in a competitive event (Douda et al., 2008), which consists of the sum of the sub-scores of all the four apparatuses of the all-around while others use the average score of several competitions (Hume et al., 1998) or the score in one apparatus (Kolarec et al., 2013). Thus, the magnitude of the variance in performance explained by the regression analysis in different studies range from 29\% (Hume et al., 1993) to 92.5\% (Douda et al., 2008), with different physical abilities identified as important. Moreover, a competitive result is multifaceted and includes different interrelated aspects of performance such as the association of movements with music, apparatus handling and the artistry of composition, that depend less on physical fitness. Therefore, for both coaches and researchers, it may be more useful to consider the separate performance scores (technical execution, artistry, difficulty), as described in the Rhythmic
Gymnastics Code of Points (F.I.G., 2013), and identify the contributing factors of physical fitness to each of those separate scores.

Childhood is considered as the best time to develop correct movement patterns for youth sports (Lloyd and Oliver, 2014) and in particular in rhythmic gymnastics, where peak performance is achieved at a relatively young age (Karpenko, 2003). Correct technical execution is at the same time a demand for success and a prerequisite for future technical development. In that aspect, physical fitness forms the basis of technical skill development in youth sports (Faigenbaum et al., 2009; Lloyd and Oliver, 2014) while a higher level of physical fitness is often related to a higher level of performance (Piazza et al., 2014; Tran et al., 2015). Therefore, the purpose of the present study was to examine the association between selected physical fitness variables and the technical execution score in young rhythmic gymnasts varying in the performance level. It was hypothesized that the two groups of rhythmic gymnasts would differ in selected physical fitness variables and the association of these variables with the technical execution score would be dependent on the athletes' level.

\section{Material and Methods}

\section{Participants}

Forty-six female rhythmic gymnasts aged $9.9 \pm 1.3$ years, who took part in the National Championships of their age category, volunteered to take part in the present study (Table 1). On the basis of the results of the individual all-around, gymnasts were divided into two groups: (a) a "qualifiers" group consisting of 24 gymnasts who ranked above the 24th place and thus qualified for the all-around, and (b) a "non-qualifiers" group consisting of 22 gymnasts who ranked below the 24th place and thus failed to qualify for the allaround. All gymnasts were free of injury, trained regularly (6 days per week of approximately 4 hours per session) and participated in competitions 3-4 times per year according to the national calendar. After a detailed briefing about the experimental procedure and the potential risks involved in the study, written parental consent was provided for all the gymnasts. The study was approved by the Institutional Review Board of the School of Physical Education and Sport Science, University of Athens, and all 
procedures were in accordance with the Declaration of Helsinki.

\section{Procedures}

All measurements were made 10-15 days after the National Championships. Physical fitness and technical execution assessments were performed during two separate sessions 4-5 days apart, starting at around $4 \mathrm{pm}$. During the first session technical execution was assessed by the same experienced, International Gymnastics Federation (F.I.G.) judge. Gymnasts performed a standardized compulsory routine of the National Gymnastics Federation, which lasted $1.5 \mathrm{~min}$ and included the basic body movements (jumps/leaps, balances and rotations). Technical execution was evaluated according to the current Rhythmic Gymnastics Code of Points (F.I.G., 2013). During the second session, anthropometric measurements were made first and then, physical fitness elements were assessed in the following order: balance, lower and upper body flexibility, muscle endurance, jumping performance, agility. Twenty-four hours prior to each session, the gymnasts were asked to avoid any strenuous activity. A standardized gymnastics warm-up (including $5 \mathrm{~min}$ jogging interspersed with general and specific movements of moderate intensity, $10 \mathrm{~min}$ of static and $10 \mathrm{~min}$ of dynamic sport specific stretching) preceded each session.

\section{Measures}

\section{Anthropometry}

Body mass and height were measured using a digital scale and a stadiometer (Seca 710, and Seca 208, Hamburg, Germany). Body composition was estimated by measuring the skinfold thickness on two sites of the body using the equation of Slaughter et al. (1988) for girls aged less than 18 years.

\section{Balance}

Static Balance was assessed by a sport-specific test used in rhythmic gymnastics (Kioumourtzoglou et al., 1997). The subjects' goal was to remain on the ball of the foot ('releve') with their arms held above their head (third position) and the free foot at a low passe (fondue) for as long as possible. Performance was recorded using a digital stopwatch. Three trials, after two practice trials, were allowed and the best result was kept for further analysis. Gymnasts were familiar with this test as they performed it in their daily practices. The intraclass correlation coefficient
(ICC) for the balance test was $0.85(\mathrm{p}<0.01)$.

Shoulder flexion and extension

Shoulder flexion was measured according to the procedures described by Sleeper et al. (2012). Prior to data collection, gymnasts' arms length was measured from the tip of the acromion to the tip of the metacarpal bone of the middle finger. Gymnasts lay in a prone position and with their shoulders flexed at $180^{\circ}$ gripped a wooden stick with the hands pronated and the thumbs touching each other. Then both arms were raised as high as possible above the ground with the stick remaining parallel to the ground and the chin touching the ground. Flexibility of shoulder flexion was determined trigonometrically, i.e. by dividing the height attained by the stick with the length of the athlete's arm and then taking the arcsine of this ratio. Shoulder extension was measured using the same procedure described above, with the gymnasts lying in a prone position and lifting their arms behind their back. The ICCs for shoulder flexion and extension tests were $0.94(p<0.01)$ and $0.97(p<0.01)$, respectively. Sit and reach

Gymnasts took a seated position with legs stretched out and feet were placed flat against a standardized sit and reach box and reached forward with both hands as far as possible, not allowing knees to flex (Pescatello et al., 2014). The ICC for the sit and reach test was $0.92(p<0.01)$. Hip range of motion

Straight leg raise range of motion of the preferred leg was measured with the gymnast lying supine on an examination bed with their lower back flat on the plinth to prevent pelvic rotation (Heyward, 2005). The preferred leg was then raised by an experienced investigator as far as possible to the point of tolerance while maintaining the knee fully extended, without the pelvis lifting off the plinth and with the ankle joint in a neutral position. The opposite leg was held firmly down by an assistant to prevent hip flexion. Markers were placed on the hip, knee and ankle joints of the preferred leg and flexibility was defined as the angle between the lifted leg and the horizontal plane using a digital camera (Casio Exilim Pro EX-F1) at 30 frames per second and Kinovea Video Analysis Software (v 0.8.15).

The sideways leg extension (developpe à la seconde) included the combined hip action of flexion, abduction and external rotation (Angioi et 
al., 2009). Gymnasts stood upright, slightly supporting one hand on the 'barre' and lifted the contralateral leg bringing it sideways and upwards as close to the trunk as possible. Both legs remained stretched and the torso was kept upright. The angle between the two legs was measured using Kinovea software, as described above. The ICCs for the straight leg raise and sideways leg extension were $0.91(p<0.01)$ and 0.96 $(\mathrm{p}<0.01)$.

Body hyperextension: The 'bridge' test was used to assess body hyperextension in rhythmic gymnastics (Rutkauskaite and Skarbalius, 2011). Traditionally, performance is measured as the distance between the wrist and the heels. However, since the height of a gymnast may affect the distance between wrists and heels the measurement was standardized by subtracting it from the gymnast's height with the arms raised (up to the wrist) and then dividing it by the height with the arms raised. The ICC for the body hyperextension test was $0.89(\mathrm{p}<0.01)$.

All flexibility measurements were made twice and the best result was used for further analysis.

Upper body muscular endurance

Muscular endurance of the arms and chest muscles was measured using the 1 min push-ups test (Ballady et al., 2000). The gymnasts were instructed to keep the body in a straight position and bend their elbows until the chin touched the mat and then fully extended their arms again. The maximum number of push-ups performed consecutively in one minute was used for further analysis. The ICC for the $1 \mathrm{~min}$ push-ups test was $0.91(\mathrm{p}<0.01)$.

The 1 min sit-up test was used to measure muscular endurance of the abdominal muscles (Barker et al., 2007). Gymnasts lay face up on a mat with their knees bent at $90^{\circ}$ and crossed their arms over their chest with hands on their shoulders at all times. From this position, gymnasts raised their upper torso until their elbows touched their knees and then lowered their upper torso until their shoulder blades touched the floor. The examiner assisted by anchoring the gymnast's feet on the ground. The maximum number of correctly executed repetitions in one minute was recorded. The ICC for the 1 min sit-up test was $0.91(p<0.01)$.

Muscular endurance of the back extensors was evaluated using a previously published test
(Trošt-Bobić and Radaš, 2010). Briefly, gymnasts lay face down and performed trunk hyperextensions to $90^{\circ}$ (from a position where the chest touched the floor to a position where the shoulder blades touched a fixed object). The maximum number of repetitions performed in 30 seconds was recorded. The examiner assisted by anchoring the gymnast's feet on the ground. The ICC for the back extension test was $0.91(\mathrm{p}<0.01)$. Jumping performance

Jumping performance was assessed by the counter movement jump (CMJ) and the drop jump (DJ) from $30 \mathrm{~cm}$ height. For the CMJ, gymnasts were instructed to perform a countermovement until the knees were bent at approximately 90 degrees, and then immediately jump as high as possible with maximal effort. For the drop jump, athletes jumped down from a 30 $\mathrm{cm}$ box onto the mat and then immediately performed a maximal vertical jump. Athletes were instructed to keep their hands on their hips throughout the test and land on the same spot. The best value of two jumps separated by $30 \mathrm{~s}$ rest was used for analysis. Jump height was calculated from flight time, using an OptoJump System (Microgate, Bolzano, Italy). The ICCs for CMJ and DJ were 0.96 and $0.95(\mathrm{p}<0.01)$ and this agreed with recently published reliability data (Markwick et al., 2015). Agility

Agility was tested with a gymnastics specific test as described by Sleeper et al. (2012). Gymnasts performed five consecutive $18 \mathrm{~m}$ shuttle sprints (running across the diagonal length of the gymnastics floor). The total time taken to run the five sprints was measured using a digital stopwatch. The ICC for this agility test was 0.91 $(\mathrm{p}<0.01)$.

\section{Statistical analysis}

Data are reported as means and standard deviations (SD). The Pearson's correlation coefficient ( $r$ ) was used to detect linear associations among selected physical fitness variables and the technical execution score. Differences between gymnasts of a higher and lower performance level were determined using independent samples t-tests. Cohen (d) effect sizes were calculated and their magnitude was categorized as follows: trivial, $<0.2$; small, 0.2 to 0.5 ; small to moderate, 0.5 to 0.8 , and large, $>0.8$ (Cohen, 1988). Multiple regression analysis was 
used to investigate which physical fitness variables contributed most significantly to the technical execution score in each group separately. Test-retest reliability for all the dependent variables measured in this investigation was determined separately by calculating the intra-class correlation coefficient (ICC) using a 2-way mixed model analysis of variance. Statistical significance was accepted at $\mathrm{p}<0.05$. All analyses were performed using SPSS (version 20.0, SPSS Inc., Chicago, IL, USA).

\section{Results}

The technical execution score was significantly higher in qualifiers compared with non-qualifiers. Qualifiers had greater hip and shoulder flexibility in most tests (Table 2). In particular, shoulder flexion, straight leg raise and sideways leg extension were significantly higher in the qualifiers ( $p<0.01$ and $d>0.82$, Table 2). Shoulder extension $(p=0.075)$ and balance on the ball of the foot $(p=0.105)$ tended to be higher in qualifiers and the effect size of the difference was moderate $(d>0.50)$.

\begin{tabular}{|lcccc|}
\hline \multicolumn{4}{c}{} & \multicolumn{2}{c|}{ Table 1 } \\
\hline \multicolumn{5}{c}{ Descriptive characteristics of the participants. Data are means \pm SD } \\
\hline & Age $(\mathrm{y})$ & $\begin{array}{c}\text { Height } \\
(\mathrm{cm})\end{array}$ & $\begin{array}{c}\text { Body mass } \\
(\mathrm{kg})\end{array}$ & $\begin{array}{c}\text { Training } \\
\text { experience }(\mathrm{y})\end{array}$ \\
\hline Non-qualifiers $(\mathrm{n}=22)$ & $9.7 \pm 1.5$ & $136.0 \pm 8.5$ & $28.3 \pm 4.0$ & $2.4 \pm 1.5$ \\
Qualifiers $(\mathrm{n}=24)$ & $10.2 \pm 1.0$ & $139.7 \pm 6.5$ & $30.2 \pm 3.6$ & $2.5 \pm 1.0$ \\
\hline & & & & \\
\hline
\end{tabular}

\begin{tabular}{|c|c|c|c|c|c|c|}
\hline \multicolumn{7}{|c|}{$\begin{array}{l}\text { Comparison of the technical execution score and physical fitness assessment test } \\
\text { results (mean } \pm S D \text { ) between Qualifiers and non-Qualifiers. } \\
\text { The correlations between the technical execution score and physical fitness } \\
\text { variables are presented separately for Qualifiers and non-Qualifiers }\end{array}$} \\
\hline Physical fitness variables & $\begin{array}{c}\text { Non-qualifiers } \\
(\mathrm{n}=22)\end{array}$ & $\begin{array}{l}\text { Qualifiers } \\
(\mathrm{n}=24)\end{array}$ & $p$ & $\begin{array}{l}\text { Effect size } \\
\text { (Cohen's d) }\end{array}$ & $\begin{array}{l}\text { Pearson's r } \\
\text { Non- } \\
\text { qualifiers }\end{array}$ & $\begin{array}{c}\text { Pearson's r } \\
\text { Qualifiers }\end{array}$ \\
\hline $\begin{array}{l}\text { Technical execution score } \\
\text { (points) }\end{array}$ & $7.77 \pm 0.56$ & $8.22 \pm 0.28$ & 0.001 & 1.05 & & \\
\hline Shoulder flexion $\left(^{\circ}\right)$ & $27.6 \pm 6.4$ & $33.5 \pm 8.4$ & 0.010 & 0.82 & 0.002 & 0.299 \\
\hline Shoulder extension $\left({ }^{\circ}\right)$ & $37.4 \pm 4.8$ & $39.9 \pm 4.6$ & 0.075 & 0.55 & $0.423^{*}$ & 0.220 \\
\hline Sit and reach $(\mathrm{cm})$ & $42.1 \pm 4.7$ & $43.1 \pm 5.4$ & 0.467 & 0.22 & 0.346 & 0.036 \\
\hline Straight leg raise $\left({ }^{\circ}\right)$ & $159.5 \pm 13.9$ & $170.2 \pm 9.9$ & 0.004 & 0.91 & 0.343 & 0.285 \\
\hline $\begin{array}{l}\text { Sideways leg extension } \\
\left(^{\circ}\right)\end{array}$ & $147.5 \pm 14.6$ & $161.1 \pm 14.0$ & 0.002 & 0.97 & $0.687^{* *}$ & $0.467^{*}$ \\
\hline Spine flexibility $(\%)$ & $84.5 \pm 8.5$ & $86.9 \pm 5.8$ & 0.262 & 0.34 & -0.158 & -0.293 \\
\hline Push ups (repetitions) & $16.5 \pm 9.6$ & $15.8 \pm 8.4$ & 0.790 & -0.08 & $0.437^{*}$ & 0.086 \\
\hline Sit ups (repetitions) & $29.5 \pm 8.7$ & $32.9 \pm 6.4$ & 0.128 & 0.47 & 0.236 & 0.226 \\
\hline $\begin{array}{l}\text { Back extension } \\
\text { endurance (repetitions) }\end{array}$ & $21.8 \pm 4.5$ & $21.7 \pm 4.0$ & 0.959 & -0.01 & 0.415 & 0.165 \\
\hline CMJ with 2 legs $(\mathrm{cm})$ & $21.0 \pm 2.2$ & $21.3 \pm 2.7$ & 0.696 & 0.12 & 0.115 & 0.241 \\
\hline $\begin{array}{l}\text { Drop jump from } 30 \mathrm{~cm} \\
(\mathrm{~cm})\end{array}$ & $21.6 \pm 3.7$ & $22.3 \pm 2.5$ & 0.474 & 0.22 & 0.033 & 0.312 \\
\hline Agility (s) & $19.8 \pm 1.9$ & $19.4 \pm 1.0$ & 0.384 & -0.27 & -0.376 & -0.186 \\
\hline Balance (s) & $10.6 \pm 12.6$ & $16.9 \pm 13.3$ & 0.103 & 0.50 & 0.273 & 0.149 \\
\hline Body fat $(\%)$ & $16.2 \pm 1.8$ & $15.0 \pm 1.4$ & 0.021 & -0.73 & $-0.642^{* *}$ & -0.078 \\
\hline \multicolumn{7}{|c|}{$* *: p<0.01, *: p<0.05$} \\
\hline
\end{tabular}




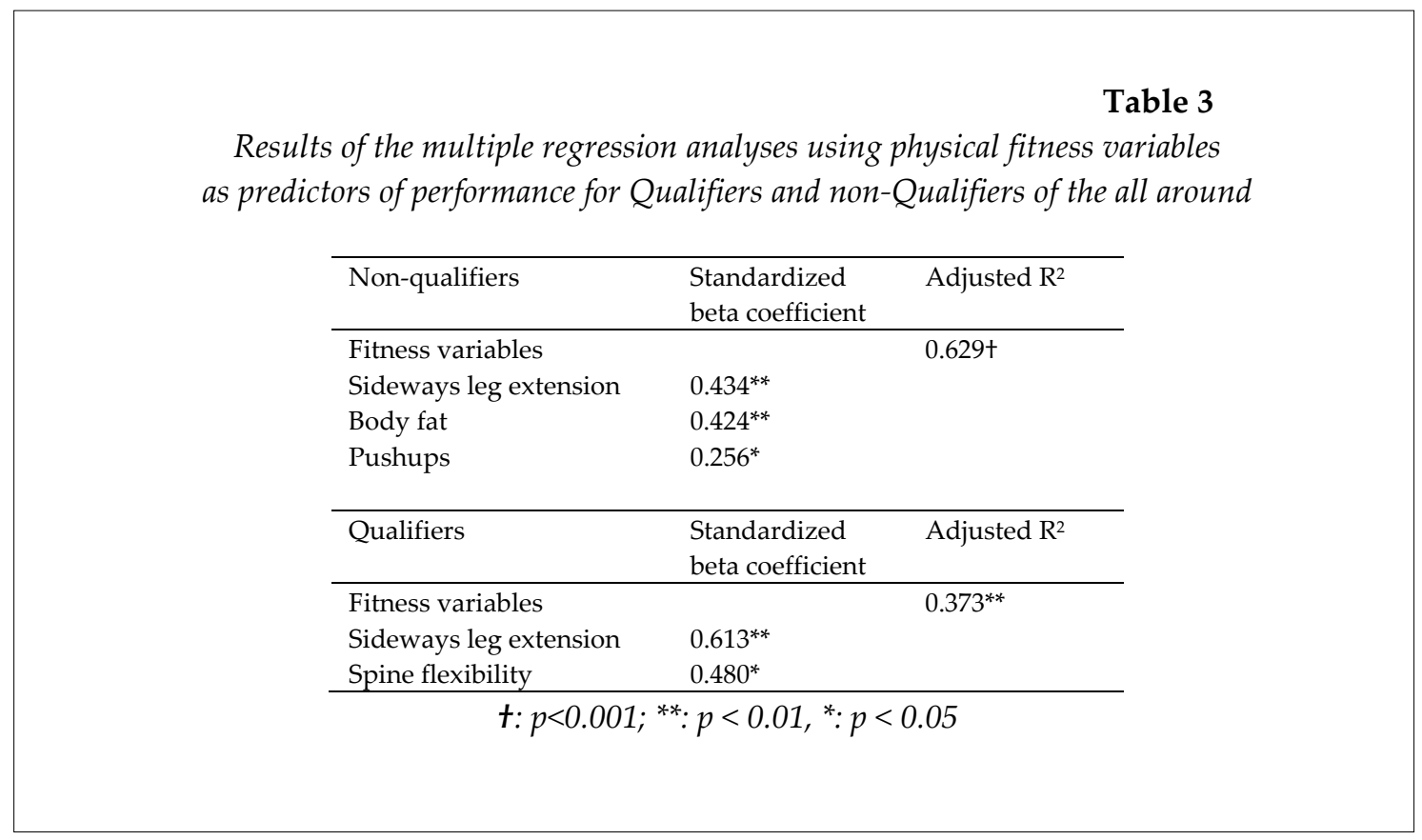

There were no other significant differences between the two groups in physical fitness variables related with muscular endurance, power (e.g. push ups, sit ups, CMJ), agility and balance, with the exception of body fat that was lower in the qualifiers compared with non qualifying gymnasts (Table 2).

The technical execution score was correlated with several physical fitness variables in the nonqualifiers, while there was only one significant correlation (between the technical execution score and sideways leg extension) for the qualifiers group (Table 2). Multiple regression analysis revealed that sideways leg extension, body fat and push ups accounted for a large part $(62.9 \%)$ of the variance in the technical execution score for the non-qualifiers, while for the qualifiers, only $37.3 \%$ of the variance in the technical execution score was accounted for by sideways leg extension and spine flexibility (Table 3).

\section{Discussion}

The main finding of the present study was that rhythmic gymnasts who qualified for the allaround differed from non-qualifiers only in the technical execution score, flexibility of the shoulder and hip joint and body composition. Interestingly, significant correlations between the technical execution score and physical fitness variables related with flexibility, upper body muscular endurance and body fat were found only in the non-qualifiers group, suggesting that within this group, those with higher physical fitness attained higher technical performance scores. On the other hand in the qualifiers group, correlations between the technical execution score and physical fitness were low and non-significant, with the only exception being sideways leg extension. The results of the regression analyses confirmed the larger contribution of physical fitness to the variance in the technical execution score in the non-qualifiers group.

Shoulder and hip flexibility significantly discriminated between qualifiers and nonqualifiers thus highlighting the importance of specific flexibility development at this age. Rhythmic gymnasts start systematic flexibility training at a young age (6-7 years old) as the age range of 7-11 years is considered a sensitive period for flexibility development (Lloyd and Oliver, 2014; Sands, 2002). The increased pliability and reduced musculotendinous stiffness of muscle tissue at this age enable greater joint range of motion to be achieved (Lloyd and Oliver, 2014). However, in the extensive schedules of young rhythmic gymnasts, it is important to identify which flexibility variables are important for successful technical execution and form the basis of further technical development. In the present study, shoulder flexion, a straight leg raise and 
sideways leg extension were found to discriminate between qualifiers and non-qualifiers while shoulder extension also tended to be higher as the effect size was moderate (Table 2). Shoulder joint range of motion is important for the manipulation of the apparatuses in an optimal distance from the body and is also necessary for all the pre-acrobatic elements with hand support. Increased hip range of motion also ensures the execution of all the basic body movements in rhythmic gymnastics, such as leaps/jumps and turns with different body configurations. Furthermore, increased shoulder and hip range of motion protects the gymnasts from the excessive load placed on the spine during "extreme" movements such as trunk hyperextension.

One interesting finding of the present study was that sideways leg extension was the best predictor of the technical execution score for both qualifiers and non-qualifiers (Table 3). Sideways leg extension includes the combined actions of hip flexion, abduction and external rotation (Angioi et al., 2009) that are necessary for leaping, balancing and turning with different body configurations. Sideways leg extension also requires firm postural control in order to properly stand while lifting and holding the leg sideways. Although in rhythmic gymnastics sideways leg extension is used in practice for training and testing static and dynamic hip flexibility, there is no data in the literature regarding its application in gymnastics. The only study that reported that sideways leg extension may be used for the assessment of specific flexibility-related skills was performed with contemporary dancers (Angioi et al., 2009). When designing a testing battery for athletes, it is important that the testing protocol is sport specific and can identify small changes in an athlete's fitness level (McGuigan, 2014). Ideally, the fitness tests should be biomechanically and physiologically specific to the sport, so that their use as an evaluation and/or selection tool is justified. In the present study, apart from the "traditional" tests used in similar studies (Rutkauskaite and Skarbalius, 2009; 2011; Radas and Trost Bobic, 2011), two novel flexibility measurements were applied, i.e. sideways leg extension and the ratio of "height to bridge". As shown in the present study, the sideways leg extension may be a very useful range of motion test for young rhythmic gymnasts and it is therefore recommended for testing and selection purposes. In addition, the ratio of "height to bridge" may provide a more accurate assessment of body hyperextension than the traditional "bridge" test as the height of the gymnast is also taken into consideration.

Although the difference in balancing on the ball of the foot between qualifiers and nonqualifiers was not statistically significant, the effect size was moderate. Given that in this study the participants were high-level young gymnasts, even borderline differences that have a moderate effect size may be "practically significant" in providing useful information for the training and selection process (Tran et al., 2015). Standing and balancing on the ball of the foot requires a correct body posture, body alignment and a stable 'releve' position. Learning how to stand comes first, while turning and leaping follows in training of young gymnasts. Since the capacity of turning includes the capacity of balancing on one leg (Karpenko, 2003), balance on the ball of the foot is a necessary skill for performing turns and rotations. Thus, it may be useful to further investigate the importance of balance on the ball of the foot to provide a basis for technical execution in young rhythmic gymnasts.

One important finding of the present study was that the technical execution score was correlated with hip and shoulder flexibility, upper body muscular endurance and body fat only within the non-qualifiers group. In contrast, in the qualifiers group, the only physical fitness variable that was correlated with the technical execution score was sideways leg extension. These findings would suggest that at a lower level of performance, more physical fitness variables may have an effect on the technical execution score. Greater importance of physical fitness at a lower level of performance was also evident in the results of the regression analyses, showing an almost two-fold larger contribution of physical fitness (hip flexibility, muscular endurance and body fat) to the variance in the technical execution score for non-qualifiers. The lower contribution of physical fitness to the variance in the technical execution score seems contradicting, since qualifiers had superior performance compared with non-qualifiers in several physical fitness tests (Table 2). However, a possible explanation may be that at a higher level of performance, where 
flexibility is developed above a certain degree, the gymnast is able to execute a technical routine with less physical effort and more elegance, so that the technical execution score depends more on finer elements of movement and less on increased flexibility (Karpenko, 2003). In the present study, qualifiers may be characterized as high-level athletes, since their technical execution score was similar to elite national standards. Interestingly, sideways leg extension was the variable that was correlated with and contributed to the variance in the technical execution score for both qualifiers and non-qualifiers (Tables 2 and 3), stressing the importance of this testing result for rhythmic gymnastics, irrespective of the performance level. The fact that lower limb muscular power and muscular endurance were not found to discriminate between qualifiers and non-qualifiers and also had no contribution to the variance in the technical execution score may be explained by the young age of the gymnasts. In contrast with flexibility that is developed at a younger age, the optimal age for improving power and strength is between 12-17 years (Lloyd and Olivier, 2014). It is thus possible that leg power may significantly contribute to performance at a later age and this warrants further investigation. In line with previous research, qualifiers demonstrated significantly lower values of body fat compared to non-qualifiers, confirming the importance of body composition as a determinant of performance in rhythmic gymnastics (Di Cagno et al., 2008; Klentrou and Plyley, 2003; Purenovic-Ivanovic and Popovic, 2014). Rhythmic gymnastics is a sport with strict demands on body mass, demanding a specific body type with a thin torso and long and thin limbs. As the level of performance improves, differences in body composition are smaller (Di Cagno et al., 2008) due to both initial selection and dietary habits.

In conclusion, hip and shoulder flexibility as well as body composition can effectively discriminate between qualifiers and non-qualifiers in youth rhythmic gymnastics, while at a lower level of performance, physical fitness seems to have a greater effect on the technical execution score. This stresses the importance of flexibility training at this age as a prerequisite for technical development. Further research should examine the physical fitness variables related to performance in older developmental ages in order to 'profile' sport specific demands and help coaches to make informed decisions regarding the training focus at different stages of athletic development.

\section{References}

Angioi M, Metsios G, Twitchett E, Koutedakis Y, Wyon M. Association between selected physical fitness parameters and aesthetic competence in contemporary dancers. J Dance Med Sci, 2009; 13: 115-123

Baker D. High-Performance Training for Sports. Human Kinetics, Champain, IL, 127-145; 2014

Ballady GJ, Berra KA, Golding LA. ACSM's Guidelines for Exercise Testing and Prescription, 9th ed. Philadelphia, Lippincott Williams \& Wilkins, 57-90; 2000

Barker R, Gledhill A, Lydon C, Miles A, Mulligan C, Saffry G, Saipe R, Sutton L. Fitness testing for sport and exercise. Harcourt Education Ltd, 259-260; 2007

Bobo Arce M, Mendez Rial B. Determinants of competitive performance in rhythmic gymnastics: a review. Journal of Human Sport and Exercise, 2013; 8: 711-727

Bogdanis GC. Effects of physical activity and inactivity on muscle fatigue. Front Physiol, 2012; 3: 142

Di Cagno A, Baldari C, Battaglia C, Brasili P, Merni F, Piazza M, Toselli S, Ventrella AR, Guidetti L. Leaping ability and body composition in rhythmic gymnasts for talent identification.J Sports Med Phys Fitness, 2008; 48: 341-346

Di Cagno A, Baldari C, Battaglia C, Monteiro MD, Pappalardo A, Piazza M, Guidetti L. Factors influencing performance of competitive and amateur rhythmic gymnastics-Gender differences. J Sci Med Sport, 2009; 12: 411-416 
Douda HT, Toubekis AG, Avloniti AA, Tokmakidis SP. Physiological and anthropometric determinants of rhythmic gymnastics performance. Int J Sports Physiol Perform, 2008; 3: 41-54

F.I.G. (Federation Internationale de Gymnastique). Rhythmic Gymnastics 2013-2016 Code of Points. International Gymnastics Federation; 2013

Faigenbaum AD, Kraemer WJ, Blimkie JR, Jeffreys I, Micheli LJ, Nitka M, Rowland TW. Youth resistance training: Updated position statement paper from the national strength and conditioning association. J Strength Cond Res, 2009; 23: 60-76

Heyward VH. Advanced fitness assessment and exercise prescription. $4^{\text {th }}$ Edition. Human Kinetics, Champain, IL, 230-240; 2005

Hume PA, Hopkins WG, Robinson D, Robinson S, Hollings S. Predictors of attainment in rhythmic sportive gymnastics. J Sport Med Phys Fit, 1993; 33: 367-377

Karpenko LA. Rhythmic Gymnastics, Russian Gymnastics Federation, St. Petersburg: Department of Physical Education, P.F. Lesgaft. 380-385; 2003

Kioumourtzoglou E, Derri V, Mertzanidou O, Tzetzis G. Experience with perceptual and motor skills in rhythmic gymnastics. Percept motor skill, 1997; 84: 1363-1372

Klentrou P, Plyley M. Onset of puberty, menstrual frequency, and body fat in elite rhythmic gymnast compared with normal controls. Br J Sports Med, 2003; 37: 490-494

Kolarec M, Horvatin-Fuckar M, Radas J. Correlation between motor skills and performance evaluation of ball routine elements in rhythmic gymnastics. Acta Kinesiol, 2013; 1: 85-89

Lloyd RS, Oliver JL. High-Performance Training for Sports. Human Kinetics, Champain, IL, 15-28; 2014

Markwick WJ, Bird SP, Tufano JJ, Seitz LB, Haff GG. The intraday reliability of the Reactive Strength Index calculated from a drop jump in professional men's basketball. Int J Sports Physiol Perform, 2015; 10: 482-488

McGuigan M. High-Performance Training for Sports. Human Kinetics, Champain, IL, 3-15; 2014

Pescatello LS, Arena R, Riebe D, Thompson PD. ACSM's Guidelines for Exercise Testing and Prescription, $9^{\text {th }}$ ed. Philadelphia, PA: Wolters Kluwer/Lippincott Williams \& Wilkins, 105-107; 2014

Piazza M, Battaglia C, Fiorilli G, Innocent G, Iuliano E, Aquino G, Di Cagno A. Effects of resistance training on jumping performance in pre-adolescent rhythmic gymnasts: a randomized controlled study. Ital J Anat Embryol, 2014; 119: 10-19

Purenovic-Ivanovic T, Popovic R. Somatotype of Top-Level Serbian Rhythmic Gymnasts. J Hum Kinet, 2014; 40: $181-187$

Radas J, Trost Bobic T. Posture in top-level Croatian rhythmic gymnasts and non-trainees. Kineziologija, 2011; 43: $64-73$

Rutkauskaite R, Skarbalius A. Interaction of training and performance of 13-14 year old athletes in rhythmic gymnastics. Ugdumas Kuno Kultura, 2011; 82: 29-36

Rutkauskaite R, Skarbalius A. Training and sport performance of 11-12 year-old athletes in rhythmic gymnastics. Ugdumas Kuno Kultura, 2009; 72: 107-115

Sands WA. Physiology, In W.A. Sands, D.J. Caine, and J. Borns. Scientific Aspects of Women's Gymnastics. Basel:Karger, 128-161; 2002

Slaughter MH, Lohman TG, Boileau RA, Horswill CA, Stillman RJ, Van Loan MD, Bemben DA. Skinfold equations for estimation of body fatness in children and youth. Hum Biol, 1988; 60: 709-723

Sleeper MD, Kenyon LK, Casey E. Measuring fitness in female gymnasts: the gymnastics functional 
measurement tool. Int J Sports Phys Ther, 2012; 7: 124-138

Tran TT, Lundgren L, Secomb J, Farley ORL, Haff GG, Seitz LB, Newton RU, Nimphius, S, Sheppard, JM. Comparison of physical capacities between nonselected and selected elite male competitive surfers for the national junior team. Int J Sports Physiol Perform, 2015; 10: 178-182

Trošt Bobić T, Radaš J. Lumbar spine dynamic stability evaluation-a new field test. Hrvat Športskomed Vjesn, 2010; 25: 75-80

\section{Corresponding author:}

\section{Gregory C. Bogdanis, PhD}

Faculty of Physical Education and Sports Sciences,

Address: 41 Ethnikis Antistasis Str, Daphne, 17237, Athens, Greece.

Tel.: +302107276115

Email: gbogdanis@phed.uoa.gr 